\title{
EFFECTIVE PERPETUAL METRICS ALGORITHM FOR VIDEO WATERMARK EVALUATION
}

\author{
Rekha B Venkatapur', Mytri V.D. ${ }^{2}$, Damodaram A. ${ }^{3}$ \\ 'Department of Computer Science Engineering A P S College of Engineering, Bangalore, Karnataka, India \\ ${ }^{2} \mathrm{P}$ D A College of Engineering, Gulbarga, Karnataka, India \\ ${ }^{3}$ Jawaharlal Nehru Technical University, Hyderabad, India \\ E- Mail : 'vb_rekha2000@yahoomail.com, ${ }^{2}$ gndecb@sanchar.net.in, ${ }^{3}$ adamodaram@jntuap.ac.in
}

\begin{abstract}
In this paper we define a new sub class of Petri nets called algebraic conservative Petri nets (ACPN) for a given symmetric group $S_{n}$. We prove that the resulting Petri net (ACPN) is a marked graph . In particular, we show that the algebraic conservative Petri nets associated with $S_{3}$ and $S_{5}$ has decompositions $=\{1,2,3,4,5\}$ and $=\left\{1,2,3,4, \ldots,{ }_{84}\right\}$ respectively, for the sets of places such that each block is both siphon and trap and hence the underlying directed graphs of these algebraic conservative Petri nets are Eulerian. Also we show that each of the ACPN associated with these groups has a subset of places which are both siphon and trap such that the input transitions equal the output transitions and both of them equal to the set of all transitions of these algebraic conservative Petri nets and hence that the underlying directed graphs of these algebraic conservative Petri nets associated with $\mathrm{S}_{3}$ and $\mathrm{S}_{5}$ are Hamiltonian.
\end{abstract}

Key words: Algebraic conservative Petri nets, siphons, traps, symmetric groups, directed graphs

\section{INTRODUCTION}

By watching what has been happening during the last few years and what is probably going to come about in the next, it seems to be clear that a novel and powerful commercial opportunity has been provided to the multimedia market. But as it happens for any marketable product, also in this case, a pressing request related to the need to safeguard goods ownership rights has been raised. Due to the ease by which digital material can be copied quickly without loss of quality, the demand for data protection has been growing unexpectedly and a way to securely defend digital documents had to be indicated. Attention has been dedicated to each one of the various media (audio [5], text [6], images [1, 3, 4], and video [2]), to succeed in embedding, separately in each kind of content, useful information regarding it. In fact in Section 2 a particular raw-video oriented application, based on a watermarking algorithm [7] originally built for still images, is presented.

\section{PROPOSED WATERMARKING TECHNIQUE}

The proposed approach consists in applying a tested and well-performing watermarking technique [7], originally devised for still images, to non-coded video, by considering it as a collection of single frames. In the following two sub sections the algorithm will be roughly described and then the main features of the approach used for video watermarking will be explained.

\section{A. Watermarking of Still Images}

The watermark casting is carried out, as described in [7], extracting the brightness of the to-be-marked frame, computing its full-frame DFT (Discrete Fourier Transform) and then taking the magnitude of the coefficients. Moreover to better preserve the original quality of the single frame, a particular masking the operation exploiting knowledge of the characteristics of the HVS (Human Visual System) is accomplished. In the detection step the luminance of the image to be checked for watermark presence is extracted and the magnitude of its DFT is considered again; only who knows the private code strings, used during the coding phase, is able to generate the exact code to look for. Furthermore resistance to geometric transformations as scaling, rotation, cropping, etc., is well-granted thanks to the insertion of a template during the coding step.

\section{B. Watermarking of Video Sequences}

Many different techniques oriented to video watermarking have been recently developed. These algorithms can be divided in two basic separate categories on the basis of which kind of video they deal with: some of them work directly in the MPEG2/MPEG4 coded domain by embedding the water-mark in the bitstream described in the previous subsection, to noncoded video sequences, processing each frame in a distinct and different way [7], is presented. In fact, thanks to the used water marking technique, the inserted code is frame-dependent and though the private key is always the same, the mark really introduced in the image is diverse every time.

\section{Robustness Evaluation}

First a trade-off between time spent for marking and the degree of robustness needed for the sequence can be achieved, in other words the lower the number of 
watermarked frames in the GOP, the faster the coding phase, but, conversely, just a minor part of the video stream will be watermarked, thus causing a robustness decrease; obviously if a superior security has to be obtained a higher amount of frames might be considered. Thanks to the good robustness the watermarking algorithm had already shown in the case of still images against usual image processing as linear/non-linear equation, noise addition, JPEG compression, etc., and geometric transformations as rotation, scaling, cropping, etc., also for video applications these longed-for characteristics are held.

\section{EXPERIMENTAL RESULTS}

The sequence is composed by frames (PAL format) of size pixels and has been watermarked and then MPEG2 coded at $6 \mathrm{Mbit} / \mathrm{sec}$ at 25 frame/sec. In Fig .1 (a) and Fig.1(b) the frame of the watermarked video and the relative detector response have been depicted respectively. The whole video is checked for the mark presence; the watermarking code is revealed when the detector response is higher than the established threshold; in Fig.1 (b) the difference between the response and the threshold is visualized, so when a watermarked frame is detected a positive spike is obtained. Going through the frames a set of periodical peaks (in this test case) is displayed.

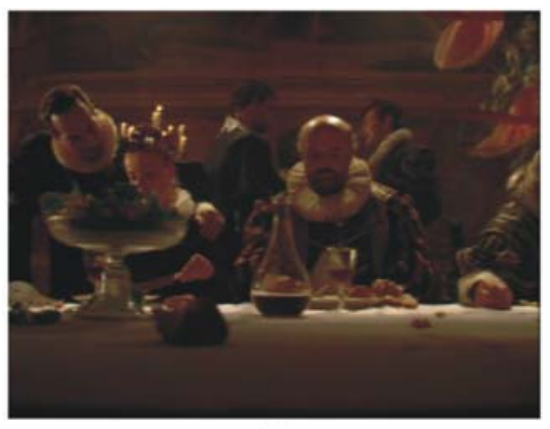

(a)

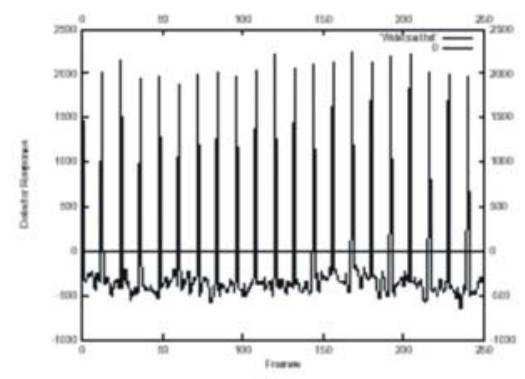

(b)

Fig .1 Test Video sequence : (a) frame $3 \mathrm{G}$ of the watermarked sequence MPEG2 coded at G Mbit/sec: (b) detector response.
In Fig .2 (a) the same frame of the test video is pictured. Now the sequence has undergone a geometrical composite attack as a degrees rotation, then an asymmetric scaling to pixels and cropping to the original size. Like the above case, the respective detector response is proposed on the right; because of the transformations occurred the spikes, though still well distinguishable, have a height lower than before and moreover two peaks in the middle of the sequence are missed, but, as explained in Section 2.3, this does not invalidate the extraction process at all. In the end, as third robustness test, another kind of attack is considered: a MPEG2 coding where bit-rate is reduced to half, in this case from $6 \mathrm{Mbit} / \mathrm{sec}$ to $3 \mathrm{Mbit} / \mathrm{sec}$

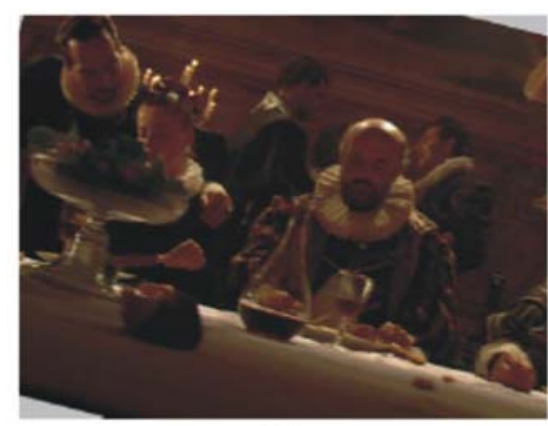

(a)

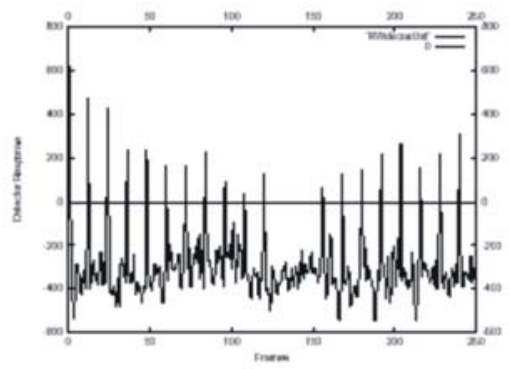

(b)

Fig .2 Test Video sequence : (a) frame $3 G$ of the watermarked sequence undergone a 15 degrees rotation. a scaling to size $1000 \times 900$ and finally a cropping to the original size :

(b) detector response.

(in Fig .3 (a) frame is depicted again). As it can be seen in Fig .3 (b) the detector is always able to exactly reveal the mark, there are no missed frames, but the height of the peaks is lower with respect to the obviously that is due to the MPEG2 coding with a decreased bit-rate. 


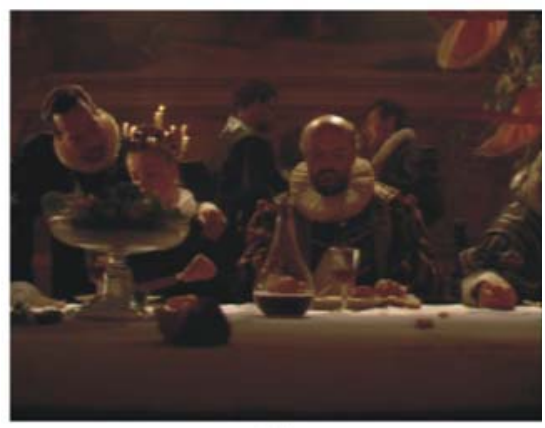

(a)

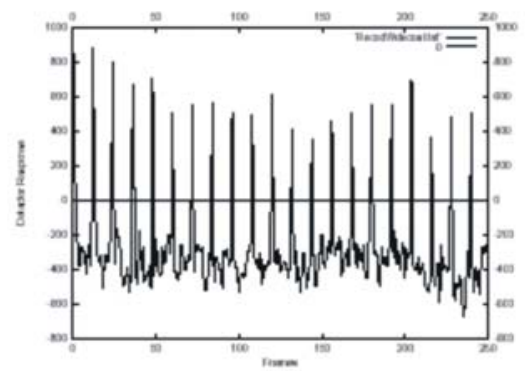

(b)

Fig .3 Test Video sequence : (a) frame $3 \mathrm{G}$ of the watermarked sequence after a MPEG2 coded at at lower bit rate (3 Mbit/sec):

(b) detector response.

\section{CONCLUSIONS}

In the present paper the attempt to apply to raw video water marking a technique initially designed only to deal with still images has been presented. Moreover, to support the goodness of this methodology, some advantages either from the point of view of the possibility of tuning the choice of which and how many frames have to be watermarked, or from the point of view of the good robustness against different sort of attacks video can be undergone, like frames exchange or frames dropping/replacing, have been highlighted. Finally experimental results confirming the effective behaviors of this technique particularly in presence of composite geometric manipulations and MPEG2 coding/decoding operations, involving bit-rate changes, have been presented.

\section{REFERENCES}

[1] M. Barni, F. Bartolini, V. Cappellini, and A. Piva, Copy right protection of digital images by embedded unperceivable marks, Image and Vision Computing, August 1998, vol.16, pp. 897-906,

[2] F. Hartung and B. Girod Digital Watermarking of Raw and Compressed Video, N. Ohta editor, Digital Compression Technologies and Systems for Video Communications, October 1996, volume 2952 of SPIE Proceedings Series, pp.205-213,

[3] M. D. Swanson, M. Kobayashi, A. H. Tewik, Multimedia data-embedding and watermarking technologies, June 1998, Proceedings of the IEEE, vol. 86, pp. 1064 \pm 1087 ,

[4] S. Craver, N. Memon, B. L. Yeo, M. M. Yeungraver, Resolving rightful ownership with invisible watermarking techniques: limitations, attacks and implications, IEEE Journal of Selected Areas in Communications, May 1998, vol4, pp. 573 \pm 586 ,.

[5] V. Basia and I. Pitas, Robust Audio Watermarking in the time-domain, September 1998, Proc. of EUSIPCO'98, Rhodes, Greece,

[6] A.K. Choudhury, N.F. Maxemchuk, S. Paul, and H.G.Schulzrinne, Copyright Protection for Electronic Publishing over Computer Networks, May/June 1995, IEEE Network, v. 9 no. 3, pp. 12-20.

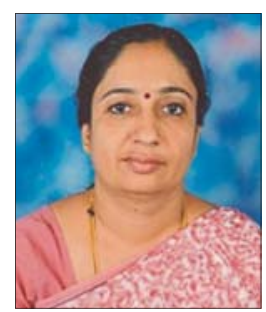

Ms. Rekha B. Venkatpur Asst. Professor Department of Computer Science Engineering, APS College of Engineering She has presented 5 papers in national and international conferences and published 3 papers in international journals. Her Conservative Petrinets. 